\title{
DiSEÑO E IMPLEMENTACIÓN DE UN SISTEMA DE CONTROL DE VOLTAJE Y FRECUENCIA DE UN SISTEMA ELÉCTRICO DE POTENCIA A ESCALA PARA LA UNIVERSIDAD POLITÉCNICA SAlesiana, SEDE Cuenca
}

\author{
IMPLEMENTATION OF A CONTROL SYSTEM OF \\ VOLTAGE AND FREQUENCY FOR AN \\ ELECTRICAL POWER SYSTEM OF SCALE OF \\ The Universidad Politécnica Salesiana \\ SEDE CUENCA
}

\author{
José Lucero $^{1}$, Jorge Nauta ${ }^{1}$, Marco Carpio ${ }^{2, *}$
}

\section{Resumen}

El presente articulo presenta la simulación e implementación de un controlador PID, mediante el uso del software MATLAB y LabView. El proceso controlado es un sistema eléctrico de potencia a escala, el cual se encuentra operativo en los laboratorios de la Universidad Politécnica Salesiana de Cuenca. El controlador descrito tiene el objetivo de mantener fijos los valores de frecuencia y voltaje producido por un generador síncrono y un motor trifásico acoplado por sus ejes mediante una banda, ante los distintos agentes externos de perturbaciones como la variación de la carga en la red eléctrica.

Palabras clave: control, frecuencia, tiempo real, voltaje, sistema eléctrico de potencia.

\begin{abstract}
This paper presents the simulation and implementation of a PID controller by using the MATLAB and LabView software. The controlled process is an Electrical Power System scale, which is operating in the laboratories of the Universidad Politécnica Salesiana of Cuenca. The controller's purpose described is to maintain fixed the values of frequency and voltage produced by a synchronous generator and a coupled three-phase motor by their shafts by a band, to various external agents disturbances such as load variation in the power grid.
\end{abstract}

Keywords: Control, frequency, real time, voltage, Electrical Power System.

\footnotetext{
${ }^{1}$ Carrera de Ingeniería Electrónica, Universidad Politécnica Salesiana, Cuenca - Ecuador.

${ }^{2, *}$ Carrera de Ingeniería Eléctrica, Universidad Politécnica Salesiana, Cuenca - Ecuador. Autor para correspondencia m mcarpio@ups.edu.ec
}

Recibido: 09-30-2016, aprobado tras revisión: 20-12-2016

Forma sugerida de citación: Lucero, J.; Ñauta, J.; Carpio, M. (2017). «Diseño e implementación de un sistema de control de voltaje y frecuencia de un sistema eléctrico de potencia a escala para la Universidad Politécnica Salesiana, sede Cuenca ». Ingenius. N. ${ }^{\circ} 17$, (Enero-Junio). pp. 60-66. ISSN: 1390-650X. 


\section{Introducción}

En los sistemas de generación eléctrica es importante mantener los valores de frecuencia y voltaje dentro de límites aceptables basados en una norma para garantizar una buena calidad de energía generada y así el correcto funcionamiento de los elementos eléctricos o electrónicos que se conecten a la red eléctrica; además, se debe poder controlar las variaciones que puede sufrir la frecuencia ante las variaciones de potencia activa o las de voltaje ante las variaciones de potencia reactiva, para lo cual existen los AVR (reguladores de voltaje automático), que son más de tipo comercial, por lo cual se propone el diseño e implementación de un controlador PID (proporcional, integral, derivativo), el cual tiene la función de mantener en un rendimiento deseado la planta (equipo o máquina que realiza una operación especifica) que controla ante posibles perturbaciones. $[1,2]$

Un sistema de control [3] se puede diseñar por diferentes métodos para los cuales son de mucha utilidad programas de computadora como Matlab para la identificación, sintonización y simulación de los sistemas de control y LabVIEW para realizar las acciones de control en tiempo real y la interacción con el usuario por medio de una interfaz gráfica y con ello manipular las variables de interés como voltaje y frecuencia.

\subsection{Sistema eléctrico de potencia a escala}

- Descripción del sistema a controlar

La etapa de generación eléctrica que forma parte de un sistema eléctrico de potencia a escala se encuentra conformada por un primotor acoplado a un generador síncrono (12 polos) mediante sus ejes por una banda, las variables a controlar son la frecuencia y voltaje generado.

El primotor dentro del sistema a escala simula la acción que provocaría un caudal que en la vida real impulsaría a las paletas presentes en una turbina, la cual es representada por el generador, esto se lo puede observar en la Figura 1. Además, el sistema a escala cuenta con el sistema de transmisión y subtransmisión.

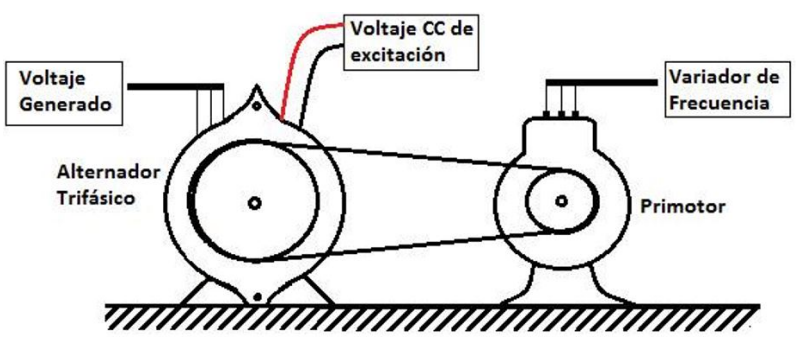

Figura 1. Planta a controlar.

\section{- Funciones de transferencia de los sistemas de frecuencia y voltaje}

Las funciones de transferencia de los sistemas fueron obtenidas mediante Matlab, de la misma manera los valores de las constantes del controlador PID. [3]

- Función de transferencia del sistema de frecuencia y constantes del controlador

Los datos con que se obtiene la función de transferencia aparecen mediante la medición de las revoluciones por minuto que se obtiene al aplicar voltaje a un variador de frecuencia que controla la velocidad de giro del primotor el cual mueve al generador, manteniendo la tensión de excitación constante. Realizando una variación de tipo escalón al voltaje aplicado se obtiene datos mediante LabView de las revoluciones por minuto los cuales son procesados mediante el comando «ident» en Matlab, obteniendo la siguiente ecuación:

$$
G(s)=\frac{1,0296}{1+1,0932 \times s}
$$

Obtenida la función de transferencia mediante la herramienta de Matlab (Sisotools) se obtendrán las constantes del controlador (KP, KI, KD), dentro de esta opción permite hacer rápido o lento, robusto o no robusto al control según las consideraciones de diseño, en este caso se obtuvieron los siguientes valores de las constantes de control para la primera función de transferencia.

$$
\begin{aligned}
K_{p} & =0,1981 \\
K_{i} & =1,0506 \\
K_{d} & =0,0212
\end{aligned}
$$

- Función de transferencia del sistema de voltaje y constantes del controlador

La función de transferencia se obtiene mediante la medición del voltaje generado y la tensión de excitación, variando esta y manteniendo constante la frecuencia. Mediante el método de los puntos se obtiene la siguiente función de transferencia.

$$
\begin{gathered}
G(s)=\frac{2,2612}{1+0,1835 \times s} \\
K_{p 2}=0,85578 \\
K_{i 2}=5,6421 \\
K_{d 2}=0,0068517
\end{gathered}
$$




\section{Simulación del controlador PID}

A continuación se procede a realizar la simulación de los controladores del sistema de frecuencia y voltaje por separado para verificar el funcionamiento y los tiempos de respuesta.

- Simulación del controlador PID con la función de transferencia del sistema de frecuencia

En la Figura 2 se presenta la simulación del controlador PID aplicado a la planta de frecuencia.

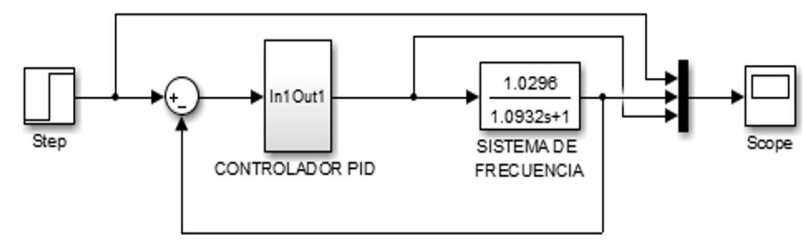

Figura 2. Simulación del controlador PID para el sistema de frecuencia.

En la Figura 3 se muestra como actúa el controlador PID (señal morada) y la respuesta de la planta ya controlada (señal verde).

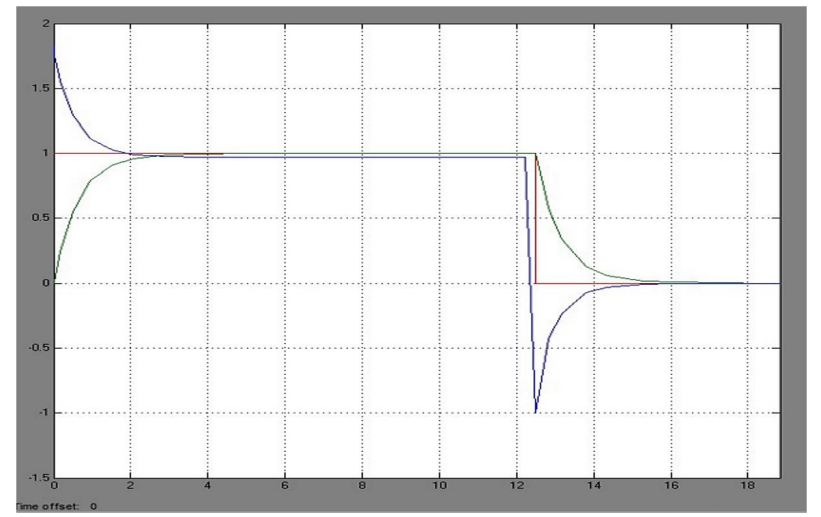

Figura 3. Respuesta del sistema de frecuencia y controlador.

Como se puede observar en la Figura 3 el tiempo de estabilización de la planta es aproximadamente de 2,5 segundos, este lapso de respuesta se puede considerar aceptable ya que el criterio en que se basa el desarrollo del controlador prioriza la precisión.

- Simulación del controlador PID con la función de transferencia del sistema de voltaje

En la Figura 4 se presenta la simulación del controlador PID aplicado al sistema de voltaje.

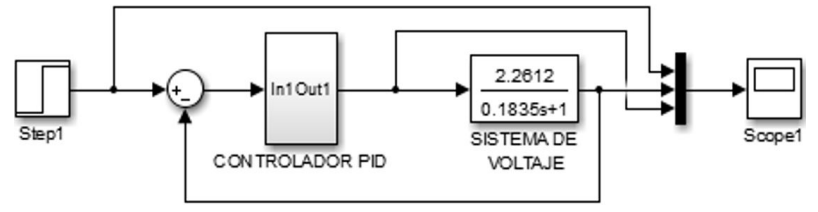

Figura 4. Simulación del controlador PID para el sistema de voltaje.

En la Figura 5 se puede observar la respuesta del controlador (señal morada) y la repuesta de la planta controlada (señal verde).

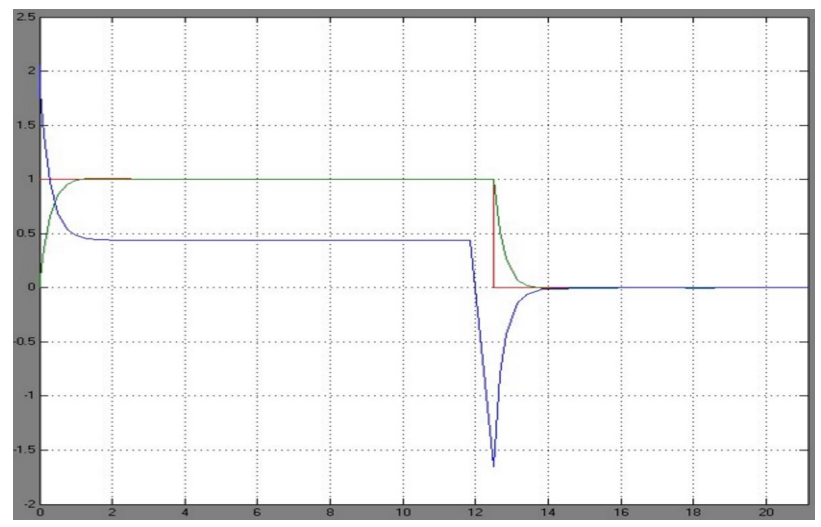

Figura 5. Respuesta del sistema de voltaje y controlador.

Como se puede apreciar en la Figura 5 el tiempo de estabilización de la planta del sistema de voltaje es aproximadamente de 1,3 segundos.

Se debe tomar en cuenta que en la vida real los tiempos de respuesta pueden variar, debido a que los dos controladores se van a relacionar.

\section{Implementación}

La implementación del sistema de control se logra mediante el uso de LabVIEW, el controlador PID se lo diseña por bloques, es decir, la acción proporcional, integral y derivativa se lo realiza por separado y se los coloca en paralelo para obtener la respuesta deseada tal como se puede observar en la Figura 6. Para la acción integral se basa en la integración trapezoidal, y para la acción derivativa se utiliza el método de diferencias finitas (diferencias hacia adelante).

Además de implementar el control, se realiza una interfaz gráfica para una interacción sencilla, tal como se muestra en la Figura 7. 


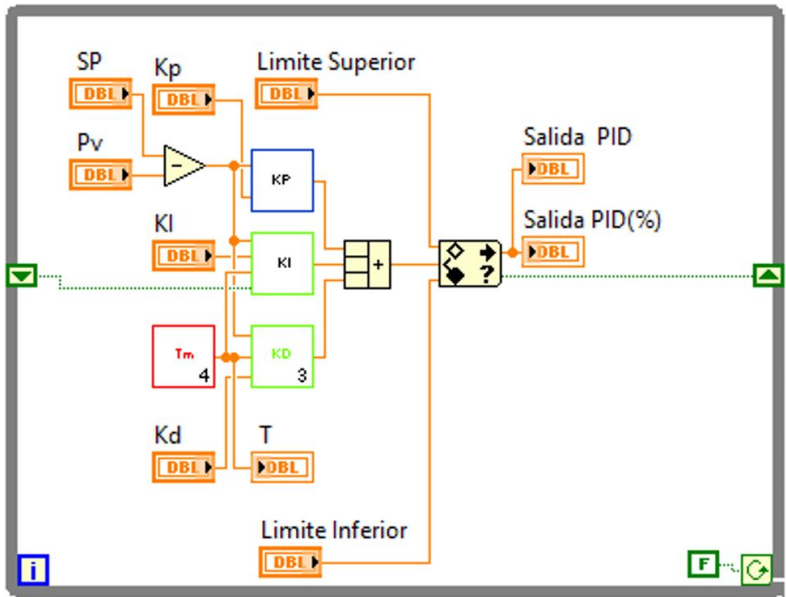

Figura 6. Implementación del controlador PID en LabVIEW.

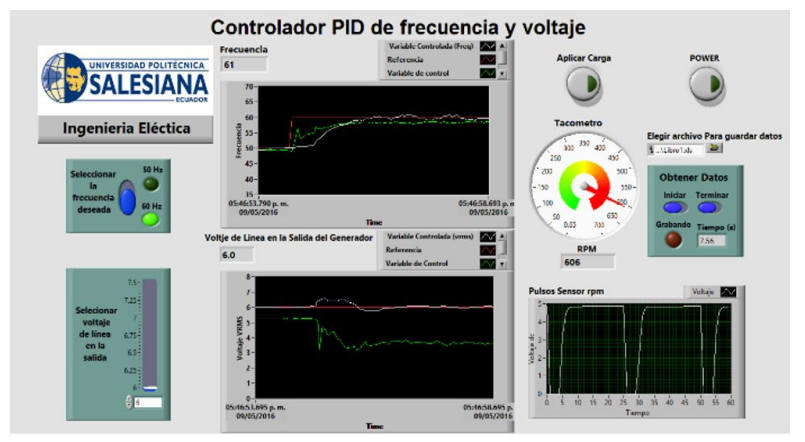

Figura 7. Interfaz del sistema de control en LabVIEW.

\section{Análisis de resultados}

En el presente bloque se indica las diferentes pruebas realizadas en LabVIEW con sus respectivos resultados analizados mediante Matlab.

\subsection{Comparación del sistema con y sin contro- lador}

- Comparación del sistema con y sin controlador ante la conexión del generador al sistema de transmisión y subtransmisión

En este apartado se muestra de manera gráfica cómo es la reacción del sistema sin controlador y con controlador, para apreciar la funcionalidad del controlador implementado en el sistema eléctrico de potencia.

En la Figura 8 que no se muestra la gráfica de la frecuencia porque la frecuencia no se afecta cuando se conecta el sistema de transmisión y subtransmisión, lo contrario sucede con el voltaje debido a que el voltaje de salida en el sistema sin controlador tiene una caída de 352 milivoltios y que se trasforman en una caída de 9,85 voltios en la etapa de amplificación, ya que el transformador elevador tiene una relación de 1:28. Además, se puede observar que durante el régimen permanente existen unas variaciones, debidas a que la adquisición de las señales de los sensores se realiza cada 60 milisegundos por motivos de procesamiento en tiempo real de las mismas.

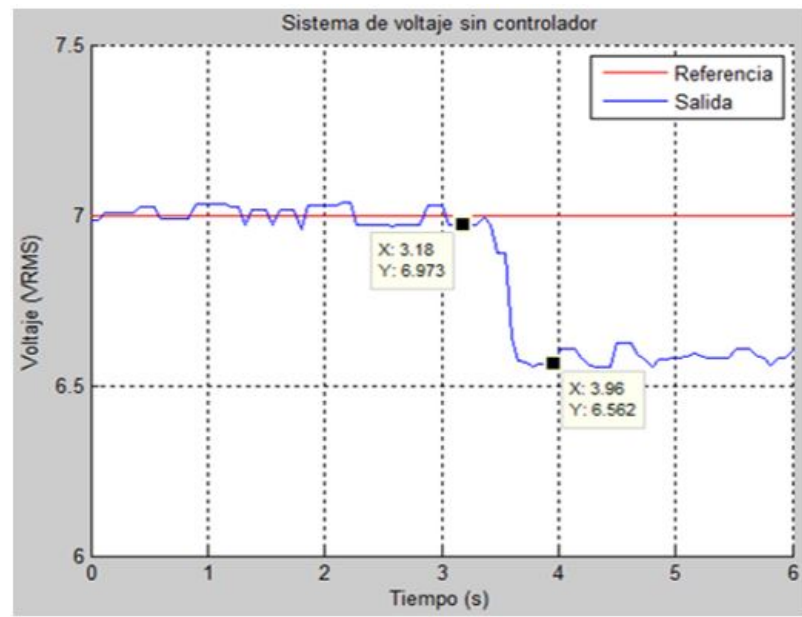

a)

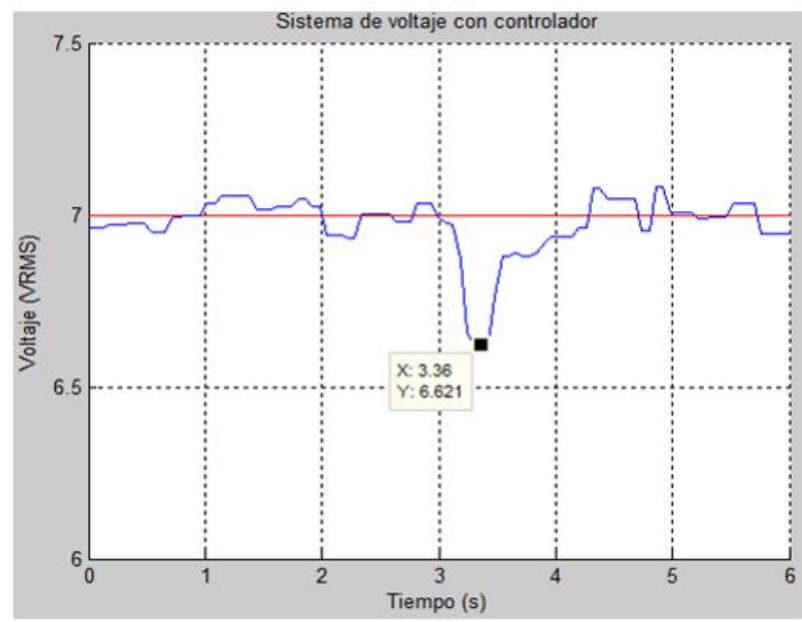

b)

Figura 8. Comparación del sistema con y sin controlador ante la conexión de carga al sistema, a) respuesta sin controlador, b) respuesta con controlador.

- Sistema sometido a un cambio en la referencia de 50 a 60 Hertz con 6 voltios en la salida

La prueba realizada a continuación muestra el desempeño del controlador, con el cual hacemos énfasis que nos basamos en la precisión más no en la velocidad de respuesta, dado que se busca no tener sobrepasos porque esto afectaría no solo al sistema de generación [4-6], sino a todos los equipos que podrían estar conectados a la red eléctrica a escala, y esto se puede observar en la Figura 9, en donde se varía la referencia de la frecuencia, cambiando de 50 a 60 Hertz. 


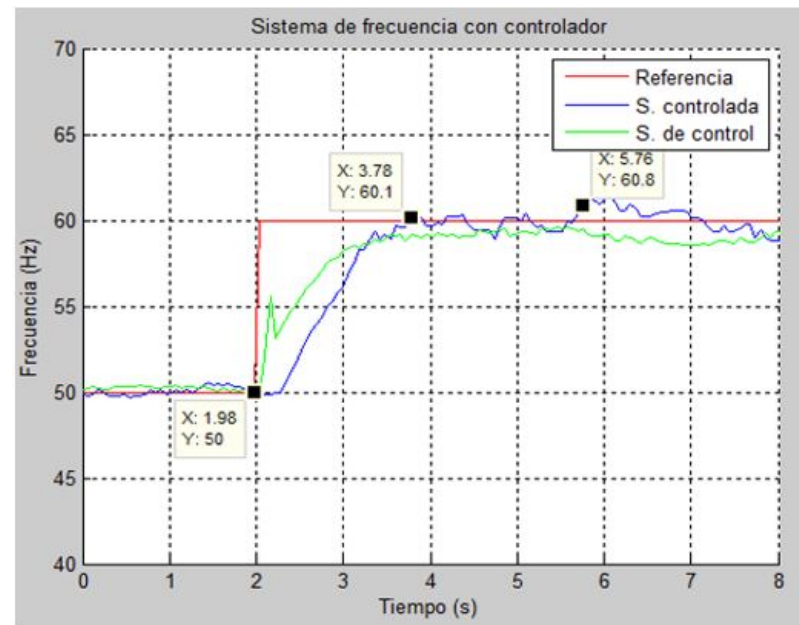

a)

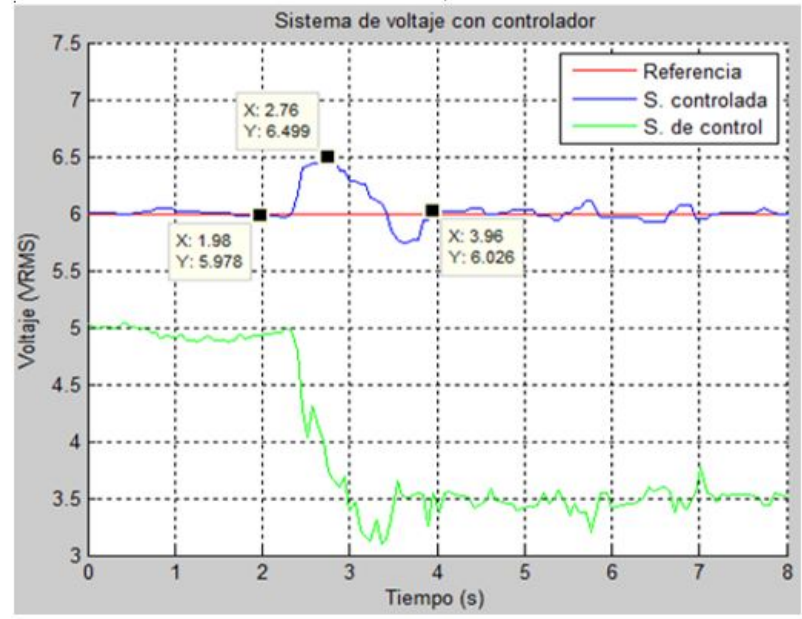

b)

Figura 9. Respuesta del sistema con controlador y sin carga ante una variación en la referencia de la frecuencia, a) respuesta de frecuencia, b) respuesta de voltaje.

En la Figura 9 se puede observar las tres señales presentes en el sistema, en donde: la curva roja es la señal de referencia, la azul es la señal controlada y la verde es la señal de control, que en el sistema de frecuencia corresponde a la conversión del voltaje enviado al variador de frecuencia, observando que es similar a la señal controlada, es decir, está por los mismos niveles, en cambio, la señal de control en el sistema de voltaje corresponde a la tensión que se manda a una etapa de potencia en donde se amplifica el voltaje en un $100 \%$ con una capacidad de corriente necesaria para la excitación del generador.

En cada sistema los tiempos de establecimiento divergen del sistema calculado, es decir, para el sistema de frecuencia el tiempo de establecimiento es de 1,8 segundos y en el sistema de voltaje es de 1,98 segundos por ello para procesar los valores medidos se basó en el tiempo de mayor duración con el cual el sistema se recupera.

El sistema de voltaje se ve un poco afectado por el incremento de la frecuencia y esto se ve reflejado en los sobrepasos de voltaje existentes, pero estos son corregidos por el controlador dentro de un tiempo aceptable según lo propuesto, dentro de este tiempo la respuesta varia aproximadamente en un rango de $\pm 8 \%$ del valor deseado.

- Sistema sometido a un cambio en la referencia de 60 a 50 Hertz con 6 voltios en la salida

En esta prueba se hace lo inverso que en la prueba anterior en relación del cambio de referencia de la frecuencia, obteniéndose lo indicado en la Figura 10.

En la comparación entre las Figuras 9 y 10 se puede observar que los tiempos de establecimiento varían, con lo cual se puede decir que el sistema se estabiliza de manera más rápida cuando se incrementa la frecuencia, asimismo, se puede notar cómo la frecuencia y el voltaje generados se correlacionan, es decir, un incremento o reducción repentina en la frecuencia provoca un incremento o decremento en el voltaje.
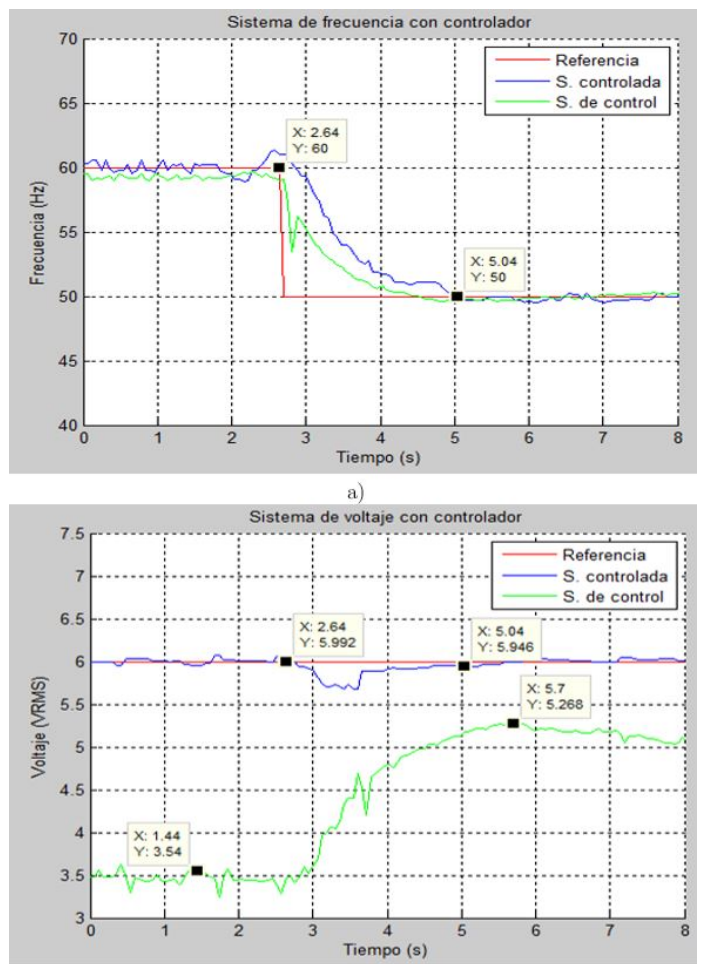

b)

Figura 10. Respuesta del sistema con controlador y sin carga ante una variación en la referencia de la frecuencia, a) respuesta de frecuencia, b) respuesta de voltaje.

- Sistema sometido a un cambio en la referencia del voltaje de salida variando de 6 a 7 voltios

A continuación, se mostrará la reacción del sistema con el controlador cuando se realiza un cambio de referencia en el voltaje [7], en donde el sistema se encuentra generando 6 voltios de corriente alterna con una frecuencia de $60 \mathrm{~Hz}$, y después de un instante se 
cambia únicamente la referencia de 6 a 7 voltios de corriente alterna, de esta manera, obtenemos lo indicado en la Figura 11.

En la Figura 11 se puede apreciar cómo la señal de control inicia con un valor aproximado de 3,5 voltios y en un instante de tiempo (120 ms) pasa a un valor máximo aproximado a 6,4 voltios, la cual sirve para compensar el incremento realizado en la referencia. La variación se la realiza a los 4,2 segundos y se obtiene un valor estable a los 5,88 segundos, esto quiere decir que el sistema se estabiliza en un tiempo de 1,68 segundos.

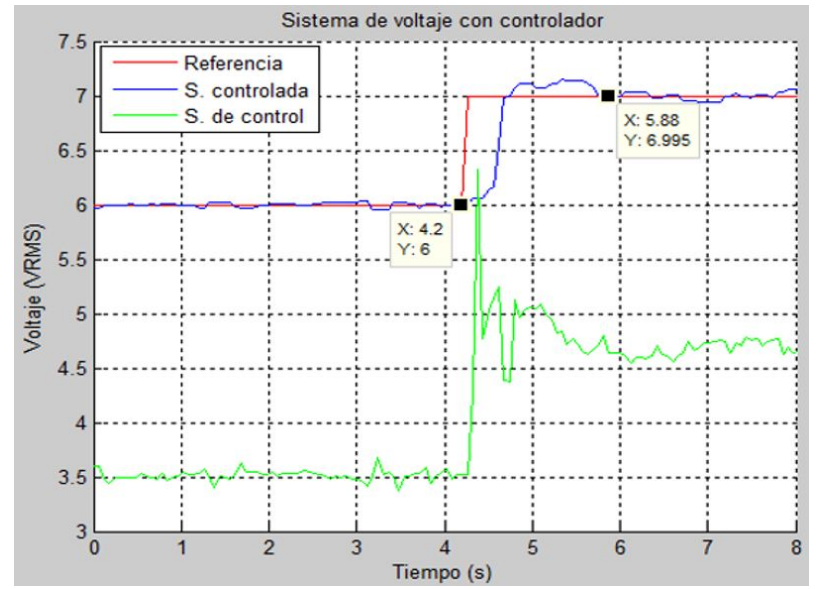

Figura 11. Respuesta del sistema con controlador y sin carga ante una variación en la referencia de voltaje.

Según los datos obtenidos se puede observar que los valores máximo y mínimo dentro de los instantes de tiempo 0 a 4,2 segundos y 5,88 a 8 segundos están dentro de valores tolerables debido a factores de adquisición, y el valor pico que tiene el voltaje hasta establecerse es de 7,15 voltios de alterna, lo que implica que el sistema se pasa de la referencia por 0,15 voltios, los mismos se reflejan como 4,2 voltios después que pasa la etapa de elevación.

- Variación de la referencia de voltaje de 7 a 6 voltios manteniendo constante la referencia de la frecuencia de 60 Hertz con carga

A continuación, en la Figura 12 se puede observar cómo el controlador se comporta ante un cambio en la referencia del voltaje deseado. En este caso el pico mínimo de voltaje es pronunciado, alcanzando el valor de 5,62 voltios de corriente alterna cuando debería estar cerca de los 6 voltios de corriente alterna, es decir, su caída de tensión está alrededor de un 6,4\%.

$\mathrm{Al}$ observar los valores obtenidos de la experimentación, el valor de la frecuencia no varía notablemente, los únicos valores críticos que existieron en esta experimentación se dieron durante el tiempo de transición que existe desde que se cambia la referencia hasta que el sistema es estable.

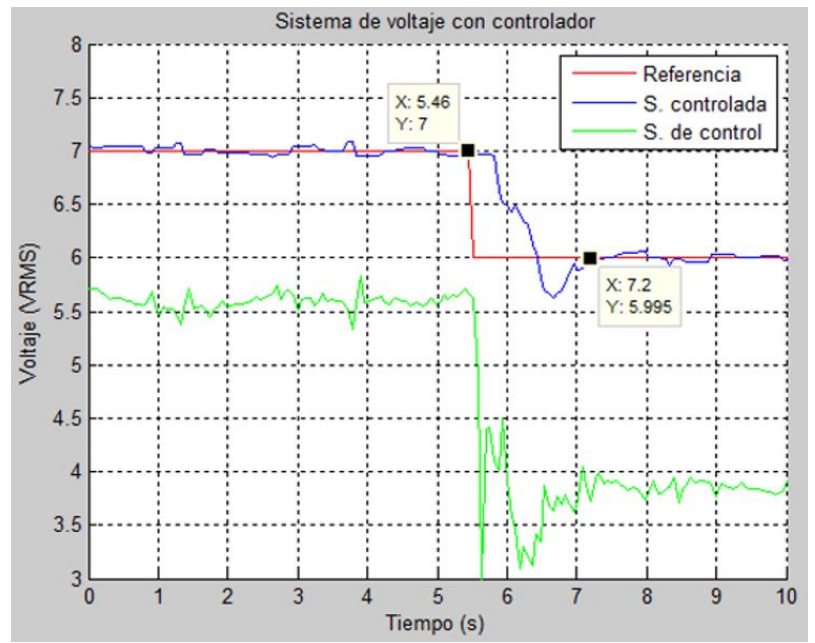

Figura 12. Respuesta del sistema con controlador y con carga ante una variación en la referencia de voltaje.

- Con disturbio en la frecuencia

A continuación, en la Figura 13 se muestra la reacción del controlador implementado en el sistema ante la introducción de un disturbio, este disturbio representa o hace analogía a una disminución crítica en el caudal en una central hidroeléctrica, por lo tanto, ante esta perturbación en nuestro caso el controlador da más tensión al variador de frecuencia para que incremente la velocidad de giro.

Según la Figura 13, ante una caída en la frecuencia de salida el controlador incrementa su señal para compensarla, lo mismo sucede en el sistema de voltaje, pero después de un instante el sistema de frecuencia llega a un pico máximo correspondiente a $64,8 \mathrm{Hertz}$, se eleva demasiado debido a la inercia propia existente en el generador.

\section{Conclusiones}

Las consideraciones para el cálculo de las constantes de control son un poco variables debido a la vibración propia de la máquina, sensibilidad y velocidad del sensor y por la correlación que existe entre el voltaje y la frecuencia; provocando la realización de ajustes pequeños en las constantes calculadas de los controladores; el ajuste realizado se basó mediante la prueba y error, tratando de obtener el mejor resultado. La velocidad de respuesta podría mejorarse si se adquiriera las señales del sensor con mayor rapidez.

El desempeño del controlador PID implementado tiene una respuesta satisfactoria a pesar de que al momento de acoplar los dos controladores aumentó el tiempo de respuesta, ya que por separado se tenía un tiempo de 1,2 segundos de estabilización y juntos aumentó a 1,8 segundos sin importar el tipo de perturbación. Una variación en la frecuencia (revoluciones 
en el generador) implica una variación considerable en el voltaje generado, en cambio, cuando existe una perturbación en el voltaje no hay cambio en la frecuencia, y por medio de las pruebas realizadas se puede comprobar cómo el controlador actúa al mismo tiempo tratando de estabilizar la frecuencia y el voltaje generado por el sistema en los valores deseados.

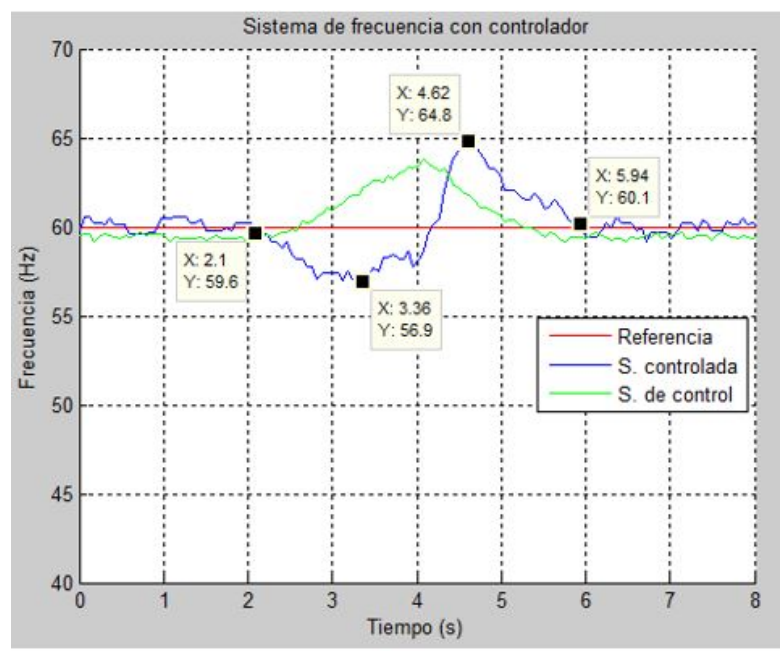

a)

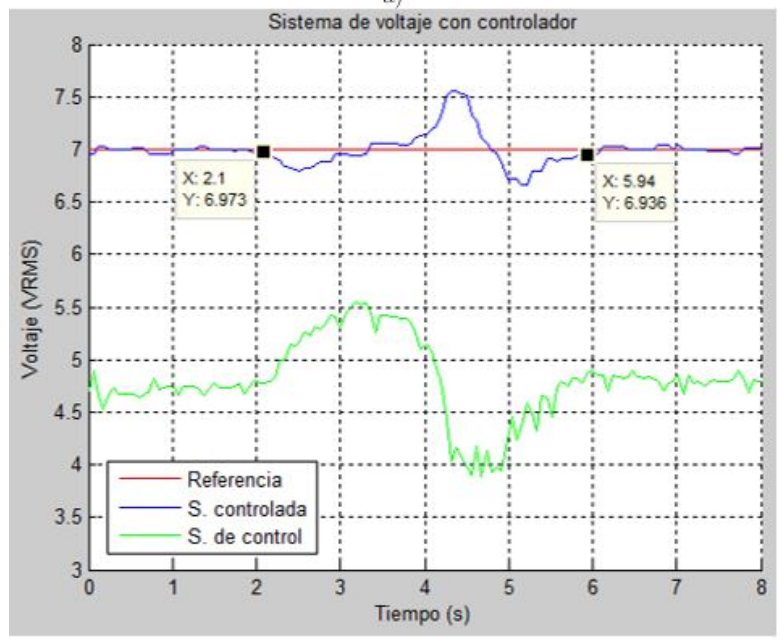

b)

Figura 13. Variación de ángulos durante el segundo ciclo de movimiento.

\section{Referencias}

[1] G. Silva, S. Datta, and Bhattacharyya, "Control System Design Using the PID Controller," pp. 191$222,2005$.

[2] A. Robles and I. Fernández, "Centrales de generación de energía eléctrica," Universidad de Cantabria, pp. 6-7, 2012.

[3] B. Kuo, Sistemas de control automático. Prentice Hall Hispanoamericana, 1996.

[4] M. Álvarez and J. González, "Diseño e implementación de un sistema de control de velocidad y voltaje utilizando Matlab," Escuela Superior Politécnica del Litoral, 2005.

[5] M. A. Ashley-Ross and G. V. Lauder, "Diseño e implementación de un sistema de control de velocidad y voltaje para la unidad 4 de generación de la central termoeléctrica Guangopolo," Universidad de las Fuerzas Armadas ESPE, 2013.

[6] M. Álvarez, J. González, and J. D. Pozo, "Diseño e implementación de un sistema de control de frecuencia y voltaje," Revista Tecnológica ESPOL, vol. 20, pp. 47-55, 2007.

[7] J. Bernal and M. Sánchez, "Construcción de un sistema de control de voltaje y frecuencia del generador síncrono Siemens, DM-SN/DG2240.Q4-R3 del laboratorio de máquinas eléctricas de la UPS, sede Cuenca," Universidad Politécnica Salesiana, 2010 . 